\title{
A Probabilistic Approach for the Generation of Learning Sessions Tailored to the Learning Styles of Learners
}

\author{
http://dx.doi.org/10.3991/ijet.v8i6.3084 \\ Jaber EL BOUHDIDI, Mohamed GHAILANI and Abdelhadi FENNAN \\ Abdelmalek Essaâdi University - FSTT, Tangier, Morocco
}

\begin{abstract}
In this paper we present an audacious solution based on Bayesian networks and educational approach for the construction of evolutionary personalized learning paths. We mean by evolutionary learning paths, paths that are composed gradually as learners advance in their learning, i.e in real time. To do this, the system selects the hypermedia units of learning to apprehend based on the results of formative assessments, psychological and cognitive characteristics of learner.
\end{abstract}

The architecture that we propose is based, firstly, on the semantic web, First, in order to model the domain model and to index learning resources so as to maximize their reuse, and then to represent the personal and cognitive traits of learners in a learner model while integrating their learning styles according to the Felder and Silverman model; and secondly, a probabilistic approach based on Bayesian networks that calculates the probability of success of each candidate hypermedia unit, for selecting those who are most appropriate for the construction of evolutionary personalized learning paths.

The proposed Bayesian model is validated with real data collected from an experimental study with a specimen of students.

Index Terms-Personalized Learning Paths, Learning Styles, Bayesian Networks, Semantic Web.

\section{INTRODUCTION}

Today, new technologies of information and communication invaded us in our workplaces, our schools and universities and more than ever necessary in all areas, namely, the field of education. These technologies have opened new access routes to knowledge. The importance of knowledge and its continuous updating throughout life requires new forms of learning. Adaptive learning systems provide a framework to respond to this challenge and ensure to learners an online training on specialized concepts and without constraints of time.

Adaptation to learner's profiles in an adaptive system of learning is a subject that worries the scientific community for a long time. The adaptation is to customize content and their presentation to the profiles of learners in order to best satisfy their needs and ensure a fast and efficient learning. The techniques related to adaptation are numerous and are based all on the learner and the domain model. Several research works are concerned. Brusilovsky et al. in [1] and [2] provides techniques and methods of adaptation based on the model of the user, Piombo [3] proposes a solution based on Bayesian networks for adaptation of learning activities, Zinber [4] proposes a service-oriented solution based on ontologies for the generation of personalized courses, Masun Nabhan [5] used Fuzzy -ART2, neural networks and fuzzy logic to provide to the learners an adaptive learning environment, Markowska-Kaczmar et al. [6] used artificial intelligence techniques to better identify the characteristics of learners in order to improve the quality of personalized learning resources.

In this paper we propose a solution based on semantic web technologies and Bayesian networks, semantic web technologies are designed to make Web resources more accessible to machines, that is to say, equip the machines capabilities to truly understand and accurately as possible the meaning of the information contained in these resources to operate effectively. On the other hand, Bayesian networks are used in many areas and have been successful in applications where there is decision making. In this solution our probabilistic model aims to make decisions about the choice of hypermedia units for the construction of evolutionary personalized learning paths.

This solution has the pretension to help learners, lamenting the difficulties or gaps, develop new concepts or improve their knowledge or expertise. Learners can use the tool outside the classroom by targeting directly the objectives to understand while taking advantage of an adaptation that takes into account their knowledge and learning styles during the generation of learning paths.

\section{APPROACH BASED ON BAYESIAN NETWORKS}

\section{A. Bayesian Networks}

Bayesian networks (BN) [7] initiated by Judea Pearl in the 1980s based on the theories of probability and graphs to represent knowledge formalism. They have been developed in several areas in the decision-making systems and expert systems, they are based on probabilities and incorporate uncertainty in their reasoning. More formally, a Bayesian network $\mathrm{B}=(\mathrm{G}, \odot)$ is defined by parameters $\odot$ $=\left\{\left(\mathrm{P}\left(\mathrm{X}_{i} \mid \mathrm{Pa}\left(\mathrm{X}_{i}\right)\right)\right\}\right.$ which is the set of probabilities for each node $\mathrm{X}_{i}$ conditional on the state of its Parents $\mathrm{Pa}\left(\mathrm{X}_{i}\right)$ in $G$, and a graph $G$ which gives a visual on the dependency relationships between the different variables of the network.

Bayes theorized that the probability of future events can be calculated according to the probabilities of their parents. Theorem Bayes states:

$$
P(A / B)=\frac{P(A \mid B) \times P(A)}{P(B)}
$$


with:

$\mathrm{P}(\mathrm{A})$ : a priori probability;

$\mathrm{P}$ (A / B): a posteriori probability;

Bayes' theorem is used to calculate the a priori probabilities for a set of variables based on the probability that other variables are supposed known in advance.

\section{B. Learning of Bayesian Networks}

The learning of a Bayesian network is the most important phase to validate it structure, in general there are two types of learning in the literature: learning of structures to find causal relationships between variables in the Bayesian network model and learning of parameters is to find the estimation of conditional probabilities.

\section{Learning of Structures}

The structure of a Bayesian network is usually defined by experts. But it is also possible to use algorithms to build the network. Amongst these algorithms there are those who are to identify the various causal relationships between variables, and others who are trying to quantify the suitability of a Bayesian network to solve the problem, that is to say: associate a score to each Bayesian network. An exhaustive approach is impossible in practice; in fact, the number of possible structures for nodes is superexponential. The following formula demonstrated by [8] gives the number of possible structures from nodes.

$$
\mathrm{NS}=\left\{\begin{array}{l}
1, \quad n=0 \text { ou } 1 \\
\sum_{i=1}^{n}(-1)^{i+1}\left(\begin{array}{c}
n \\
i
\end{array}\right) 2^{i(n-1)} N S(n-1), \quad n>1
\end{array}\right.
$$

Several heuristics to solve this problem have been proposed in the literature [7]. Among these methods are: Algorithm PC (causal search) Tree maximum weight, the greedy search (GS).

\section{Learning of Parameters}

The learning of parameters is an approach that involves estimating the conditional probability tables of variables. For this, two methods can be used depending on the state of the database, either from complete or incomplete data. At first, if all variables are observed, the easiest method and most used is the statistical estimate, which is to estimate the probability of an event by the frequency of occurrence of the event in the database. This approach, called maximum likelihood gives us then [7].

$$
P\left(X_{i}=x_{k} \mid p a\left(X_{i}\right)=x_{j}\right)=\odot_{i, j, k}=\frac{N i, j, k}{\sum_{k} N i, j, k}
$$

Where $\mathrm{N}_{i, j, k}$ is the number of events in the database for which the variable $\mathrm{X}_{i}$ is in the state $\mathrm{X}_{k}$ and his parents are in $\mathrm{X}_{j}$ configuration.

In a second time, in practical applications, databases are often incomplete. Some variables are observed only partially or never. Many methods attempt to estimate the probability density of the missing data. Consider the case of the Expectation Maximization algorithm (EM) [7] which is one of the most widely used algorithms for learning in $\mathrm{BN}$.

\section{APPROACH BASED ON LEARNING STYLES OF LEARNERS}

Learning styles are one of the individual differences that play an important role in learning. The learning style means anything that is characteristic of an individual when he learns, ie the specific approach to a learning task, the activated learning strategies during performance of a task. Several definitions of learning styles have been proposed in the literature, we retain its of Beshuizen et al. "A predisposition on the part of some students to adopt a particular learning strategy regardless of the specific requirements of the learning task"[9].

Learning styles can be seen as cognitive styles that learners prefer to store and process information. Numerous studies have shown the importance of taking into consideration the students learning styles in the adaptation of learning sessions. Indeed, the interest of researchers in the field of learning styles was born several models of learning styles [10]: Kolb model, Honey and Mumford model, Felder and Silverman model... etc.

In this article, we used the Felder and Silverman model. The Felder and Silverman model classifies learners according to the means they use to collect information and process that information. In this model, the preferences of students are classified into four dimensions, the following table summarizes the classifications of learning styles by Felder and Silverman:

TABLE I.

FELDER AND SILVERMAN LEARNING STYLE MODEL

\begin{tabular}{|c|l|}
\hline Learning Style & \multicolumn{1}{|c|}{ characteristic } \\
\hline Active/Reflective & $\begin{array}{l}\text { Active : retain and understand information } \\
\text { best by discussing it, applying it or explaining } \\
\text { it to others; prefer group work; } \\
\text { Reflective: retain and understand information } \\
\text { best by thinking about it first; prefer working } \\
\text { alone; needs thinking time during lectures. }\end{array}$ \\
\hline Sensing/Intuitive & $\begin{array}{l}\text { Sensing : like to learn facts; tend to be more } \\
\text { practical and careful; do not like courses that } \\
\text { have no apparent connection to the real world } \\
\text { Intuitive: prefer discovering possibilities and } \\
\text { relationships ; like innovation and dislike } \\
\text { repetition; tend to work faster and are more } \\
\text { innovative but may be careless }\end{array}$ \\
\hline Visual / Verbal & $\begin{array}{l}\text { Visual : remember best what they see, (e.g. } \\
\text { pictures, diagrams, demonstrations); } \\
\text { Verbal: gets more out of words, either written } \\
\text { or spoken explanations. }\end{array}$ \\
\hline Sequential/Global \\
\hline $\begin{array}{l}\text { Sequential: gain understanding in small } \\
\text { sequential, logical steps; } \\
\text { tend to follow logical stepwise paths while } \\
\text { problem solving; may not understand material } \\
\text { fully but are still able to solve problems and } \\
\text { pass tests. } \\
\text { Global: seem to learn in large jumps, } \\
\text { absorbing material almost randomly without } \\
\text { seeing connections, then suddenly "getting it"; } \\
\text { may be able to solve complex problems } \\
\text { quickly, or put things together in a novel way } \\
\text { once they have grasped the big picture; may } \\
\text { have difficulty in explaining their knowledge; }\end{array}$ \\
\hline
\end{tabular}

\section{APPROACH BASED ON SEMANTIC WEB}

Introduced in 2001 by Berners-Lee, the Semantic Web responds to web 2.0 in developing approaches and techniques to machine not only store information but to understand their meaning in order to give users what they want in such a way unambiguous [11]. Currently, the machines do not have to blindly manipulate information, but they must perceive the data they process in order to facilitate the communication between computer systems 
and users. For this, the machine must not only have access to the information used by the human being but also the semantics associated with them. The semantic web is the core technology of Web 3.0 and has come to give to machine the dimension of perception the semantic of information processing. With Semantic Web technologies, machines can collect and process data available on the web to ensure a better use.

Ontologies [11] are one of technologies of the Web Semantic the most prevalent in the field of knowledge management; they aim to represent knowledge so as to be perceptible to the human and the machine. In this paper, we exploited the semantic web technology to represent the characteristics of learners, educational resources and teaching approaches to ensure effective exploitation during the construction of personalized learning paths. Ontologies that we designed within the framework of the solution plays a crucial role in the mapping between the intentions of learners and profiles of hypermedia units. We have designed a number of ontologies to manage all information used by the architecture. Let's start with the ontology of goals.

\section{A. Ontology Of Objectives}

The ontology of objectives defines a classification of educational objectives that are used, by learners to formulate their intentions and hypermedia units to specify the objectives they seek to achieve. An objective is defined by its type and a subject taught in the field and can be measured by a degree of control (very low, low, average, good, very good). We chose Bloom's taxonomy [12] to classify the objectives of the field taught. Bloom's taxonomy is organized into six categories "knowledge", "understanding", "application", "analysis", "synthesis" and "evaluation". This organization requires a linear exploration levels, ie, to achieve the objectives of a higher level must meet the objectives of the level which is directly below, for example, to achieve the level of analysis it must first meet the objectives of applicationlevel and so on. Figure 1 gives a graphical representation of the ontology of goals using the concepts of OWL namely the concepts of class, ObjectProperty, DatatypeProperty, ...etc.

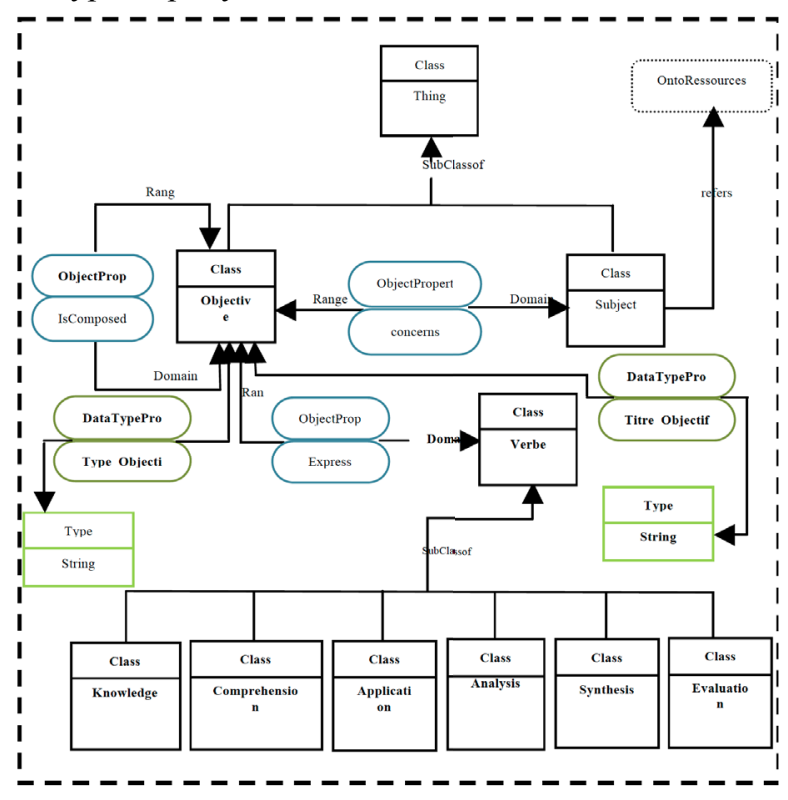

Figure 1. Ontology of Objectives Based on Bloom's Taxonomy

\section{B. Ontology Of Learning Resources}

To remedy the problems of re-use, accessibility and interoperability of learning objects, we have developed an ontology used to define the terminology for the description of educational resources. We chose to decomposing each chapter into several units hypermedia, hypermedia unit represents the finer level of granularity of contents may be reused in several courses. Each unit responds to an operational objective and may take several forms, audio, video, text, image ... etc. To index and provide context to use hypermedia units, we defined three levels of description based on the LOM standard [13]. These levels are used to represent the all information hypermedia unit, including the teaching method the most adequate for its implementation, the figure 2 shows an overview of the ontology.

\section{Ontology Of Learners Profiles}

This ontology defines the learner profiles by modeling their preferences, goals, skills and learning styles. To design this ontology, we adopted the standard IMS-LIP [14], we expanded it's to include the learning styles of learners according to the Felder and Silverman model.The Felder and Silverman model accepts the characteristics of learners in terms of learning styles, according to four dimensions:

i. Perception: Sensory / Intuitive

ii. The preferred format: Visual / Hearing

iii. Participation: Active / Reflective

iv. Presentation of content: Sequential / Global

This ontology is used by the system for the mapping between the demands of learners and profiles of hypermedia units to best meet their needs. It is also used to update the profiles of learners at the end of each training session or following a request of the student if his performance is modest. The figure 3 shows an overview of the ontology of learner profiles.

\section{THE ADAPTATION MODEL}

We come to the heart of our system, in this section we will focus on the presentation of the principles of adaptation and dynamic composition implemented by the proposed solution for generating personalized learning paths. The adaptation model intelligently imitates the work of teachers in planning learning sessions respecting preferences, learning styles and goals of learners.

For this, the adaptation model applies a number of techniques to respond to requests of learners. These techniques are applied after decomposition of the formulated objective to operational objectives.

\section{A. Adaptation According to the Learning Styles of Learners}

Our solution incorporates in its model adaptation, an adaptation according to the learning styles of learners. In a first time, and for their first authentication, learners must respond carefully to questionnaire of Felder and Silverman to communicate their learning styles. The system stores the responses of learners to identify their learning styles for use during the construction of personalized learning paths. Adapting the learning paths according to the learning styles, is used to determine the a format of hypermedia units the most preferred by the learner and the number of exercices, the number of exam- 
A Probabilistic Approach to the Generation of LeARning Sessions TAilored to the LeARning Styles...

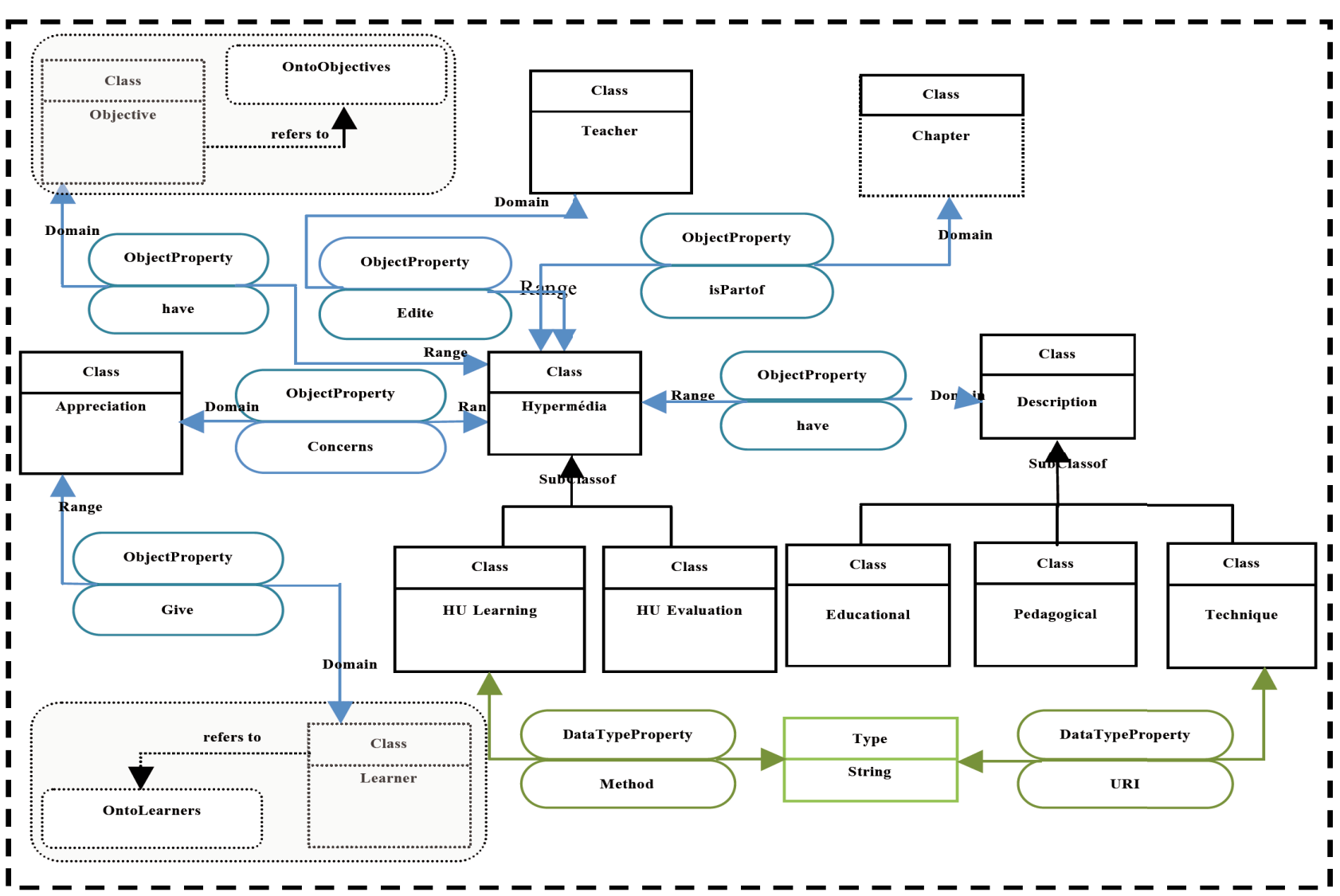

Figure 2. Ontology of Resources

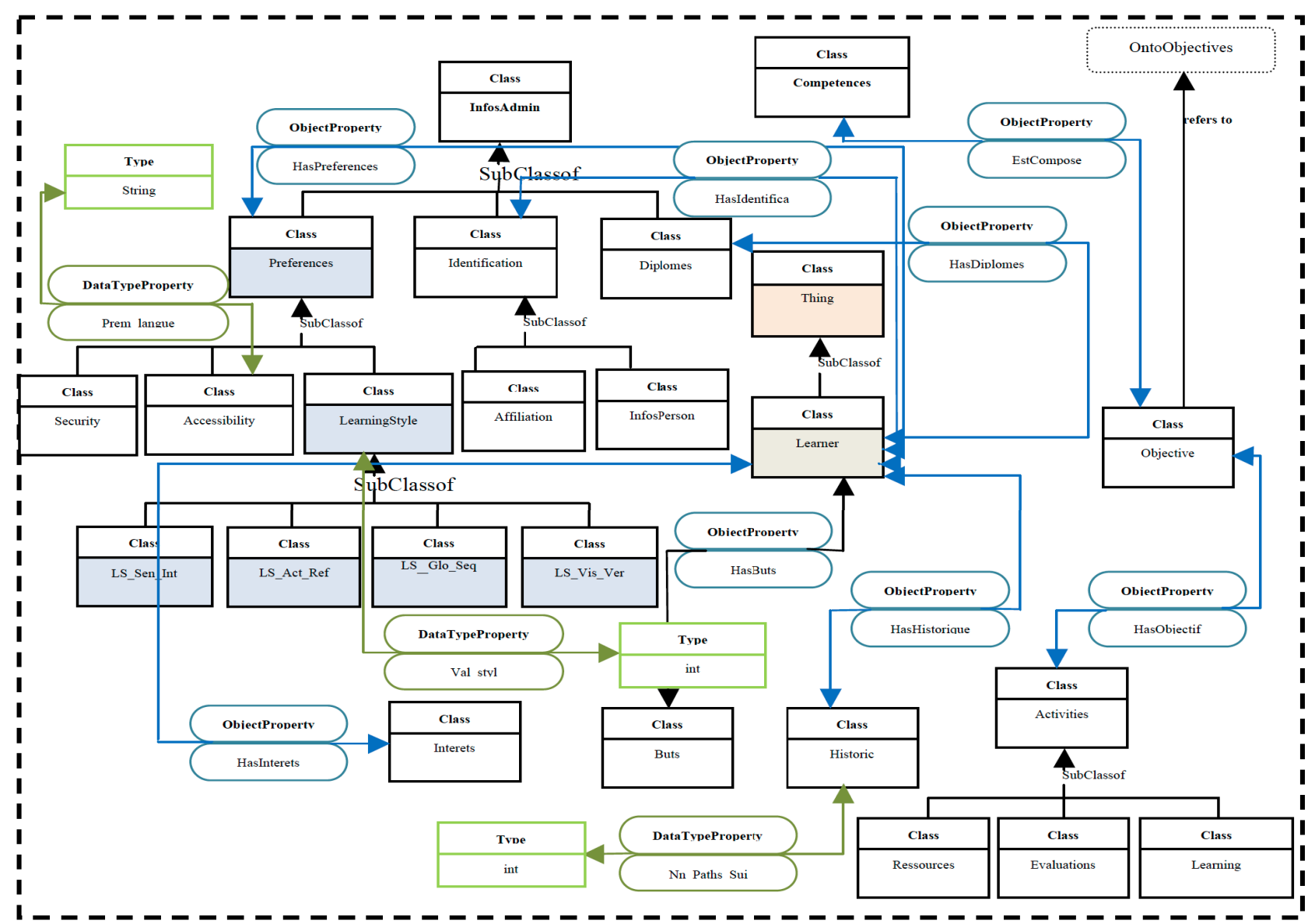

Figure 3. Ontology Of Learners Based on IMS-LIP 
ples and the position of the summary that may include the learning paths. To determine the preferences of learners in terms of number of exercises, examples ... etc.. We based on the study that was conducted by Graf [15] showing the relationship between the parameters and learning styles.

\section{B. Adaptation According To The Cognitive State Of The Learner}

The proposed solution is based on approach of pedagogical by goals, formulating intentions of learners as objectives is already a customization in itself because first, the educational criteria that channel its formation are expressed unnoticed and then, part of the educational criteria is explicitly expressed. Indeed, the learner directly target concepts to apprehend when choosing appropriate targets. It is therefore, a personalization guided by goals. Thing that facilitates the task for the learner and the system, because only hypermedia units responding to the objective formulated will be returned and then passed to the cognitive filter.

This second adaptation technique is to apply the rules of adaptation related to the cognitive state of the learner. Adaptation by cognitive status consists in calculating the degree of mastery of pre-required concepts before exposure learning contents. To calculate the degree of mastery of the knowledge, the system uses the following formula:

$$
\begin{aligned}
& \quad \operatorname{DM}(\mathrm{C})=\sum_{1}^{n} \frac{D M(O i)}{n} \text {, with } \operatorname{DM}\left(O i_{i}=\sum_{j=1}^{m} \frac{D M(S O j)}{m}\right. \text { and DM } \\
& \in[0 . .1] \\
& \text { with, }
\end{aligned}
$$

DM (C): Degree of mastery of the competency C;

$\operatorname{DM}\left(\mathrm{O}_{i}\right)$ : Degree of mastery of the objective $\mathrm{O}_{i}$ component competence;

$\mathrm{DM}\left(\mathrm{SO}_{j}\right)$ : Degree of mastery of the sub-objective $\mathrm{SO}_{j}$ component the objective $\mathrm{O}_{i}$, if $\mathrm{O}_{i}$ is decomposable.

The obtained value of $\mathrm{DM}(\mathrm{C})$ or $\mathrm{DM}\left(\mathrm{O}_{i}\right)$ is interpreted as follows: DM between 0 and 0.2 or 0.3 and 0.4 , the level of pre-required knowledge in this case corresponds respectively to very low and low. The system proposes to the learner the activities to fill gaps before him are presented learning course asked.

DM between 0.4 and 0.6 , between 0.7 and 0.8 or between 0.9 and 1, the level of pre-required knowledge in respectively medium, good and excellent. The system chosen in this case, and in so far as possible, the hypermedia units whose level of difficulty is in the vicinity of the pre-requisite knowledge of the learner.

The system then performs a matching between the level of pre-required knowledge and the difficulty of HUs to choose the most appropriate HUs. After the selection of all hypermedia units meet all the operational objectives component the objective formulated by the learner, it is necessary to choose for each operational objective the hypermedia unit who is more appropriate. To do this, the system uses a Bayesian model that calculates for each set of hypermedia units embodying the same operational objective, the probability of success of each depending on the characteristics of the learner to choose the most likelihood of success high.

\section{Adaptation Learning Paths Using a Bayesian Model.}

So far, adaptation techniques we used in our solution can indeed adapt learning paths to the learner profiles.
However, several learning paths can be generated. The system must choose the nearest to the profile of the learner. To do this, we propose a Bayesian model for creating evolutionary learning paths. Evolutionary learning paths are generated progressively as learners advance in his career, ie, in real time and take into account the results of intermediate tests taken by the learner, learning style and degree of control of knowledge required, for pre-selection the most appropriate next hypermedia unit. This is a technique that aims to help the system to make decisions on the choice of the most appropriate hypermedia units and suddenly approaching the maximum possible to the learner profile.

This refinement of the learning paths based on a Bayesian model allows to the system to take more minutely the knowledge of learners and avoids the generation of several learning paths. Let's start by identifying variables and the structure of the Bayesian model. Then, we present the results of validation of this model.

\section{Structure of Bayesian Model}

The Bayesian model that we propose is a causal network, his structure is defined based on discrete and continuous variables representing the characteristics of the learner, the results of intermediate tests and metadata hypermedia units.

Learner characteristics: Characteristics of learners that we have taken into account for the choice of the most appropriate hypermedia unit are:

Learning styles of learners are represented by four discrete random variables $\mathrm{S}=\{\mathrm{S} 1, \mathrm{~S} 2, \mathrm{~S} 3, \mathrm{~S} 4\}$. Each variable represents the projection of the learner on one of the axes of the Felder and Silverman model (VisualVerbal, Sequential-Global, Reflective-Active, SensoryIntuitive). Each variable $\left\{\mathrm{S}_{i}\right\} \quad \mathrm{i}=[1,4]$ is an integer between -11 and $+11 . S_{i} \in[-11,-9,-7,-5-3,-1,1,3,5,7,9,11]$ $; \forall \mathrm{i} \in\{1,2,3,4\}$.

- The result of the formative evaluation (intermediate test),that occurs after each operational objective, is stored in a continuous random variable $\mathrm{FE} \in[0 . .20]$.

- The degree of mastery of pre-required knowledge is represented by a continuous variable $\mathrm{DM} \in[0 . .1]$.

Hypermedia units characteristics: The hypermedia units are characterized by educational and pedagogical features that allow them to distinguish themselves from each other. In our Bayesian model, we selected the four heavy characteristics of hypermedia units, represented by four discrete random variables $\{\mathrm{HU}=\mathrm{HU} 1, \mathrm{HU} 2, \mathrm{HU} 3$, HU4\}:

- The HU1 variable represents the format of the hypermedia unit. It can take three values $U H 1 \in\{T, A$, V\} (T) text illustrated with pictures and / or diagrams (A) Audio with graphics and / or drawings of illustration and (V) Video or Animation accompanied by textual explanations.

- The HU2 variable reprents the level of difficulty of the hypermedia unit. It can take five values $H U 2 \in\{\mathrm{VE}, \mathrm{E}$, $\mathrm{M}, \mathrm{D}, \mathrm{VD}\}$, with $\mathrm{VE}=$ Very Easy, $\mathrm{F}=$ Easy, $\mathrm{M}=$ Medium, $\mathrm{D}=$ Difficult and VD = Very Difficult.

- The HU3 variable represents the appropriate teaching method to exposure the hypermedia unit. It can take 
three values $\mathrm{HU} 3 \in\{\mathrm{E}, \mathrm{A}, \mathrm{I}\}$, with $\mathrm{E}=$ Expositive, and $\mathrm{A}=$ Active $\mathrm{I}=$ Interrogative.

- HU4 the variable represents the number of examples involving the hypermedia unit. It can take a value between HU4 [0 .. n].

In addition, each learning objective may not have any concept prerequisites, as it can have several. Pre-required knowledge directly influence the acquisition of new knowledge. The figure 4 shows the relationship the degree of influence between mastery of pre-required knowledge, learning styles of learners and formative evaluations on the success of hypermedia units.

The problem consists in selecting among all candidates hypermedia units of learning that the probability of success is the highest. To do this, we must calculate the probability of success of all HULs candidates and choose the most appropriate HUL ${ }^{\text {appro }}$, ie, the one who is the probability of his success is the highest. The calculation of this probability is based on the following parameters: the degree of mastery of prerequisite objectives, results of formative evaluations (FE) and learning styles of learners. Degrees of mastery of all pre-required objectives and result in FE upstream the objective $\mathrm{OO}_{j}$ have a causal influence on the acquisition of this objective $\left(\mathrm{OO}_{j}\right)$. In other words, if the level of pre-required knowledge is good, and the results of formative evaluations are satisfactory, the probability that the learner successful the objective is high.

$\mathrm{HUL}^{\text {appro }}=\operatorname{argmax}\{\mathrm{P}($ Success $\mid\{\mathrm{HU} 1, \mathrm{HU} 2, \mathrm{HU} 3, \mathrm{HU} 4\},\{\mathrm{FE}, \mathrm{DM}\}\}$

The probabilities that we need to specify in the network of figure 5 are the a priori probabilities of nodes with no parent: master's degrees pre-required knowledge, formative evaluations, learning styles and the probability distribution conditional variables associated with different parent nodes.

$\mathrm{P}\left(\mathrm{S}_{\mathrm{i}}\right) \mathrm{i}=1, \ldots, 4$ probability of learning styles.

$\mathrm{P}(\mathrm{FE})$ Probability of formative evaluations.

$\mathrm{P}\left(\mathrm{HU} \mathrm{U}_{\mathrm{i}} \mid \mathrm{DM}\right)$

$\mathrm{P}\left(\mathrm{HU}_{\mathrm{i}} \mid \mathrm{FE}\right)$

$\mathrm{P}\left(\mathrm{HU}_{i} \mid \mathrm{S}_{j}\right) \forall j \in[1 . .4]$

$\mathrm{P}(\mathrm{HUi} \mid \mathrm{DM}, \mathrm{EF}, \mathrm{S} 1, \mathrm{~S} 2, \mathrm{~S} 3) \forall i \in[1 . .4]$

P(Success|HU1, HU2, HU3, HU4,DM,FE)

\section{Calculations and validation of the model}

When a student valid his operational objective, it should be to select among all candidates hypermedia units, fulfilling the following operational objective, which is more appropriate. This is the role of Bayesian model that calculates the probability of success of all hypermedia learning units and choose the highest. First, a phase of discretization of continuous variables in our Bayesian model is essential, subsequently, we started the phase to estimate the initial probabilities and conditional probability densities (CPD). These probabilities are calculated from the training examples or can be given by domain experts. To assign the initial probabilities to the variables of our Bayesian model, we adopted an experimental approach with sample of 101 students divided into three groups (GR1.35 students) (GR2. 34 students) and (GR3. 32 students). We first collected their learning styles using the questionnaire Felder and Silverman. Then, we proposed learning activities and assessment of different levels of difficulty and different formats. We then measured the degree of success for each learning activities based on the characteristics of learners and those of hypermedia learning units. Some data were incomplete, such as learning styles, students have not answered all the questions, which sometimes makes the calculation of the learning styles impossible, some results were strange, very good pre-required with results that are too weak. Table II shows a sample of the data obtained, we worked on a reduced set of variables to facilitate the calculation:

TABLE II.

EXTRACT FROM THE EMPIRICAL RESULTS

\begin{tabular}{|c|c|c|c|c|c|c|c|}
\hline UH2 & UH3 & DM & EF & S1 & S2 & S3 & Success \\
\hline VE & 0 & B & M & Active & Visual & Intuitive & Y \\
\hline E & 1 & M & B & Active & Verbal & Sensoriel & Y \\
\hline D & 2 & B & M & $*$ & Visual & Sensoriel & Y \\
\hline VD & 1 & B & T & Reflexive & Visual & Intuitive & N \\
\hline D & 0 & TB & M & Reflexive & Verbal & Intuitve & Y \\
\hline VD & 0 & F & M & Active & $*$ & Sensoriel & $*$ \\
\hline M & 1 & M & F & $*$ & Verbal & Intuitive & Y \\
\hline
\end{tabular}

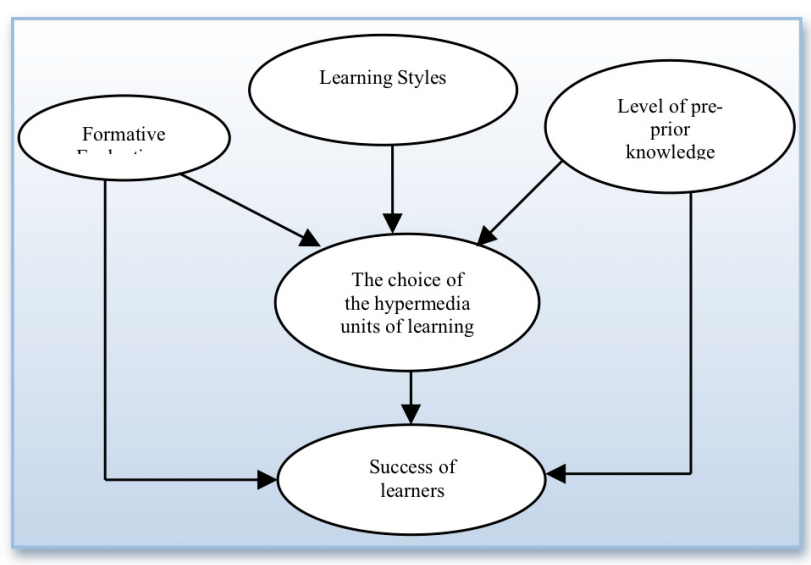

Figure 4. Causal relationships that influence the learners

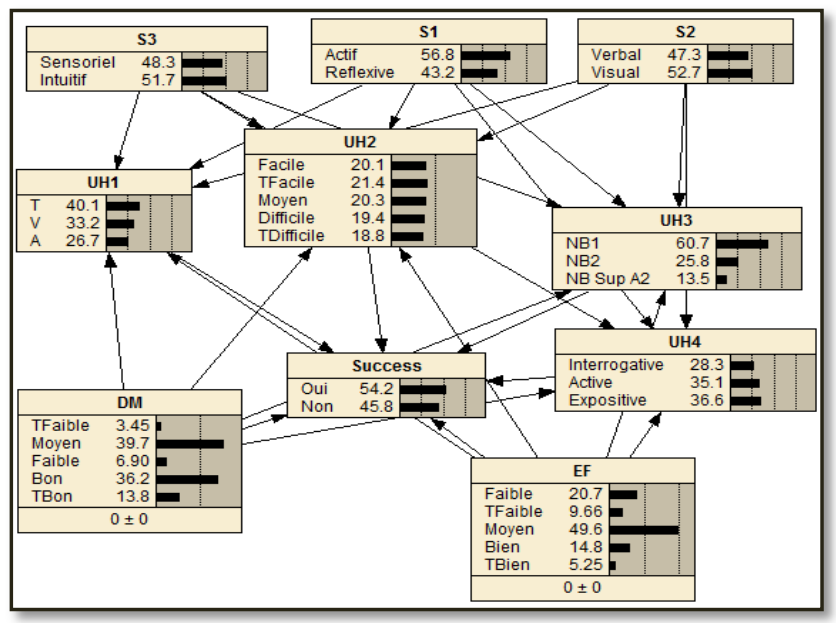

Figure 5. Structure of our Bayesian model 
Method most used for the estimation of probability distributions in a Bayesian network from complete data method is the statistical estimate. This is to estimate the probability of an event by the occurrence of the event in the database. This approach is called maximum likelihood (ML). She then gives us:

$P\left(X_{i}=x_{k} l p a\left(X_{i}\right)=x_{j}\right)=\bigodot_{i, j, k}=\frac{N_{i, j, k}}{\sum_{k} N_{i, j, k}}$

Where $\boldsymbol{N}_{\boldsymbol{i}, \boldsymbol{j}, \boldsymbol{k}}$ is the number of events in the database for which the variable $\mathrm{X}_{i}$ is in the state $\mathrm{X}_{k}$ and his parents are in $\mathrm{X}_{j}$ configuration.

However, another method can be used to estimate the probability distributions in the case where the data are incomplete. In most cases the databases are partially incomplete. It is then necessary to choose the most appropriate to estimate the missing data method. These can be estimated by using the EM algorithm (Expectation Maximization) [16], [7]. EM uses the iterative optimization method to estimate the unknown parameters $\odot^{*}$, based on measured variables $\mathrm{K}$ and data hidden $\mathrm{J}$. The idea is to maximize the a posteriori probability of the unknown parameters (marked with *) knowing $\mathrm{K}$ while marginalizing $\mathrm{J}$.

$$
\odot^{*}=\operatorname{argmax} \sum_{j \in S} P(\odot, J \mid K)
$$

The data obtained are used to calculate the parameters of each node in the Bayesian network. We used the EM algorithm to estimate the a priori probabilities and missing data. For validation of our Bayesian model (Figure 6) we used $80 \%$ of the data for training and $20 \%$ for validation.

The following figure shows the performance of the EM algorithm for estimating missing data and initialization tables of conditional probabilities.

\begin{tabular}{|rll|}
\hline Doing EM Learning, hold down & <ctrl> + <alt> \\
Iteration & Log Likelihood & Change \\
0 & 10.7053 & \\
1 & 8.12471 & 24.1061 \\
2 & 8.10754 & 0.2113 \\
3 & 8.10145 & 0.0751 \\
4 & 8.09581 & 0.0696 \\
5 & 8.09183 & 0.0491 \\
6 & 8.08979 & 0.0252 \\
7 & 8.08888 & 0.0112 \\
8 & 8.0883 & 0.0071 \\
9 & 8.08799 & 0.0038 \\
10 & 8.08787 & 0.0016 \\
11 & 8.08779 & 0.0010 \\
12 & 8.0877 & 0.0011 \\
13 & 8.08759 & 0.0014 \\
14 & 8.08745 & 0.0017 \\
15 & 8.08733 & 0.0015 \\
16 & 8.08727 & 0.0007 \\
17 & 8.08725 & 0.0003 \\
18 & 8.08723 & 0.0002 \\
19 & 8.08722 & 0.0002 \\
20 & 8.08721 & 0.0001 \\
21 & 8.0872 & 0.0001 \\
22 & 8.08719 & 0.0001 \\
23 & 8.08719 & 0.0001 \\
24 & 8.08718 & 0.0001 \\
25 & 8.08718 & 0.0000 \\
& & \\
\hline
\end{tabular}

Figure 6. Running the EM algorithm to estimate the density of missing data
A probabilistic inference can be performed in the network to calculate the posterior probabilities for different variables of hypermedia units and then infer the probability of success. Several inference algorithms for Bayesian networks have been developed include a few: the algorithm "Message Passing" algorithm "junction tree JLO" algorithm and "cut-conditionning set" of Jensen ... etc. We used the algorithm "Message Passing" applied to junction trees of maximal cliques. Hypermedia unit corresponding to the maximum probability of success will be selected. In addition, we use a continuous reinforcement of the network for new cases observed are used to reinforce the Bayesian model parameters.

\section{CONCLUSION AND FUTURE WORKS}

In this paper we have presented a solution based on Bayesian networks and the Semantic Web for the construction of evolutionary personalized learning paths. In a first time, we designed several ontologies to represent the information used by the architecture. First, the ontology of learners to represent their profiles based on standard IMS-LIP while integrating their learning styles according to the Felder and Silverman model, ontology of resources is designed on the basis of standard LOM to index hypermedia units of learning and assessment, and then the ontology of educational objectives that can represent the objectives of a training module according to Bloom's taxonomy. In a second time, we developed a Bayesian model based, firstly, on cognitive and psychological characteristics of students and, secondly, the pedagogical and educational features of hypermedia units to calculate the probability of success of the last and choose the highest.

As a perspective of this work, we plan to use other artificial intelligence techniques such as neural networks and the algorithm of $\mathrm{K}$ Nearest Neighbor (KNN) to generate personalized learning paths and thus be able to compare their results with the network Bayesian.

\section{REFERENCES}

[1] Brusilovsky P., Peylo C. Adaptive and intelligent Web-based educational systems. In P. Brusilovsky and C. Peylo (eds.), International Journal of Artificial Intelligence in Education 13 (24), Special Issue on Adaptive and Intelligent Web-based Educational Systems, pp.159-172, 2003.

[2] Peter Brusilovsky, Eva Millán, User models for adaptive hypermedia and adaptive educational systems, Springer-Verlag, 2007.

[3] Piombo C.; « Modélisation probabiliste du style d'apprentissage et application à l'adaptation de contenus pédagogiques indexés par une ontologie ». Thèse de doctorat en informatique. Université de Toulouse - Institut National de Polytechnique, 2007.

[4] ZNIBER Najlaa, «Modèle orienté service pour la conception de parcours pédagogiques personnalisés », Thèse de doctorat, Université Paul Cézanne - Faculté des Sciences et Techniques de Saint-Jérôme, 2009.

[5] Masun Nabhan Homsi Jubrini , Intelligent WEB-Based Interactive System for Education, Thèse de doctorat ,University of alepdo Faculty of science, Syrie, 2010.

[6] Markowska-Kaczmar U., Kwasnicka H., Paradowski M. Intelligent Techniques in Personalization of Learning in eLearning Systems. Studies in Computational Intelligence, Computational Intelligence for Technology Enhanced Learning, Vol. 273, 2010.

[7] P. LERAY. Réseaux Bayésiens : Apprentissage et Modélisation des Systèmes Complexes. Département ASI, INSA de Rouen, 2006. 
PAPER

A Probabilistic Approach to the Generation of LeARning Sessions TAilored to the LeARning Styles...

[8] R. ROBINSON. Counting unlabeled acyclic diagraphs. In C. Little, editor, Combinatorial Mathematics V., 1977. http://dx.doi.org/10.1007/BFb0069176

[9] Beshuizen J.J. et E.T. Stoutjesdijk. Study Strategies in a Computer Assisted Study Environment. Learning and Instruction, 1999. http://dx.doi.org/10.1016/S0959-4752(98)00027-9

[10] Popescu Elvira , Dynamique Hypermedia System for e-Learning , Thèse de doctorat Université de Technologie Compiègne, Novembre 2008.

[11] Bourhis Antoine et Perrin Nastassia, Etat de l'art sur les Technologies du Web sémantique, 2010.

[12] Bloom Benjamain; "Taxonomie des objectifs pédagogiques ». T1. Le domaine cognitif. Presses de l'Université du Québec. 1975.

[13] IEEE LOM Standard for Learning Object Metadata http://standards.ieee.org/findstds/standard/1484.12.1-2002.html, dernière consultation, mai 2013.

[14] IMS Global Learning Consortium Inc., « Learning Design Specification » http://www.imsproject.org/learningdesign/, 2003

[15] Sabine Graf, Ph.D. Thesis 'Adaptivity in Learning Management Systems Focussing on Learning Styles'. 2007.

[16] McLachlan, G. et Krishnan T., the EM algorithm and extensions. Wiley series in probability and statistics. Jhon Wiley \& Sons, 1997.

[17] Bert Chen, Chen-Yu Lee and I-Chang Tsai, "Ontology-Based ELearning System for Personalized Learning”, International Conference on Education, Research and Innovation, IPEDR vol.18 (2011) (C) (2011) IACSIT Press, Singapore.

[18] Amal Zouaq, Dragan Gasevic and Marek Hatala, «Towards open ontology learning and filtering », Information Systems Elsevier 2011.

[19] Firas ABOULATIF, « identification du profil des utilisateurs d'un hypermédia encyclopédique à l'aide de classifieurs basés sur des dissimilarités », Thèse de doctorat de 1'ENSA de Rouen, 2011.
[20] M.J. Cox, Formal to informal learning with IT: research challenges and issues for e-learning, 2012. http://dx.doi.org/10.1111/j.1365-2729.2012.00483.x

[21] Nadjet ZEMIRLINE 'Assisting In The Reuse Of Existing Materials To Build Adaptive Hypermedia', Thèse de doctorat de l'université PARIS-SUD 11, 2011.

\section{AUTHORS}

Jaber EL BOUHDIDI, is Ph.D. student in LIST Laboratory at Abdelmalek Essaadi University. His research interest includes semantic web Bayesian Networks, Neural networks, and Multi-agents Systems in E-Learning. He has several papers in International Conferences and Journals. He is a Teacher in CPGE-Rabat Morocco. (jaber.f15@gmail.com)

Mohamed GHAILANI, is a Ph.D student in LIST Laboratory at Abdelmalek Essaadi University. His current research interest includes semantic web, and Multi-agents Systems in E-Learning. He has several papers in International Conferences and Journals. He has more than Six years of Teaching Experience, and he is actually a professor of computer sciences and the ICT for Education at the Regional Center for the professions of education and training (CeRMEF) of Tangier - Morocco. (ghalamed@gmail.com)

Abdelhadi FENNAN, is Ph.D doctor and professor of computer science at Faculty of Sciences and Technology of Tangier -Morocco. He is part of many boards of international journals and international conferences. He has published several articles (afennan@gmail.com).

Submitted 31 July 2013. Published as re-submitted by the authors 08 November 2013. 